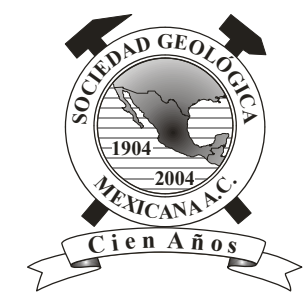

\title{
Análisis palinológico del Cenozoico de la cuenca de México: el registro polínico de los pozos Texcoco-I y San Lorenzo Tezonco
}

\author{
Socorro Lozano-García ${ }^{1, *}$, Susana Sosa-Nájera ${ }^{1}$ \\ ${ }^{1}$ Instituto de Geología, Universidad Nacional Autónoma de México, Ciudad Universitaria, delegación Coyoacán, 04510, D.F., \\ México. \\ *mslozano@unam.mx
}

\section{Resumen}

Se presenta el registro palinológico de muestras provenientes de los pozos profundos Texcoco-I (2065 m) y San Lorenzo Tezonco (2008 m) de la Cuenca de México. Los conjuntos del Plioceno-Pleistoceno de ambas secuencias se caracterizan por la presencia de elementos templados como Pinus, Quercus, Liquidambar y Picea entre otros. Para estas edades, hay evidencias en ambos sitios de la existencia de un cuerpo de agua con base en el registro de Pediastrum y Botryococcus. En los sedimentos del Texcoco-I hay registro de taxa templados para el Oligoceno, la composición del conjunto de palinomorfos es distinta con presencia de Bursera, Carya, Liquidambar y Myrtaceae. La ausencia de polen de elementos templados y la presencia de anhidrita caracterizan el paleoambiente del Eoceno tardío.

Palabras clave: Palinología, Cenozoico, Sedimentos lacustres, Centro de México

\begin{abstract}
We present the palynological record of Texcoco-I $(2065 \mathrm{~m})$ and San Lorenzo Tezonco $(2008 \mathrm{~m})$ deep wells from the Basin of Mexico. The Pliocene-Pleistocene assemblages of both sequences are characterized by temperate taxa Pinus, Quercus, Liquidambar and Picea. Microfossils of Pediastrum and Botryococcus provide evidence that the basin hosted a lake. Although temperate taxa is present in the Oligocene Texcoco-I sediments the general pollen assemblage is distinct, with Bursera, Carya, Liquidambar and Myrtaceae. The absence of temperate elements along with the presence of anhydrite the late Eocene environment.
\end{abstract}

Keywords: Palynology, Cenozoic, lake sediments, Central Mexico

\section{Introducción}

La cuenca de México es una de las cuencas intermontanas de la Faja Volcánica Transmexicana (FVTM) formadas por la actividad tectónica y volcánica asociada a la subsidencia de las placas de Cocos y Rivera desde el Oligoceno. Localizada en el sector este de la FVTM, es una cuenca de altura $(2240 \mathrm{msnm})$ con un área de $9600 \mathrm{~km}^{2}$. En el fondo de la cuenca se encuentra la planicie lacustre, donde por los procesos de subsidencia se acumuló un importante espesor de sedimentos lacustres; hay varias subcuencas sedimentarias, de norte a sur: Zumpango y Xaltocan al norte, Tecocomulco en el noreste, Texcoco al centro y Xochimilco y Chalco al sur.

La cuenca está limitada por un conjunto de sierras volcánicas, al norte por la sierra de Pachuca, al oeste por Las Cruces y al este por la Sierra Nevada donde se localizan importantes elevaciones como el Popocatépetl (5465 msnm) y el Iztaccíhuatl (5230 msnm). El límite al sur está constituido por el campo volcánico del Chichinautzin el cual divide las cuencas de México y Cuernavaca (Figura 1). Las primeras edades de 0.78 a 0.79 Ma obtenidas para el evento 


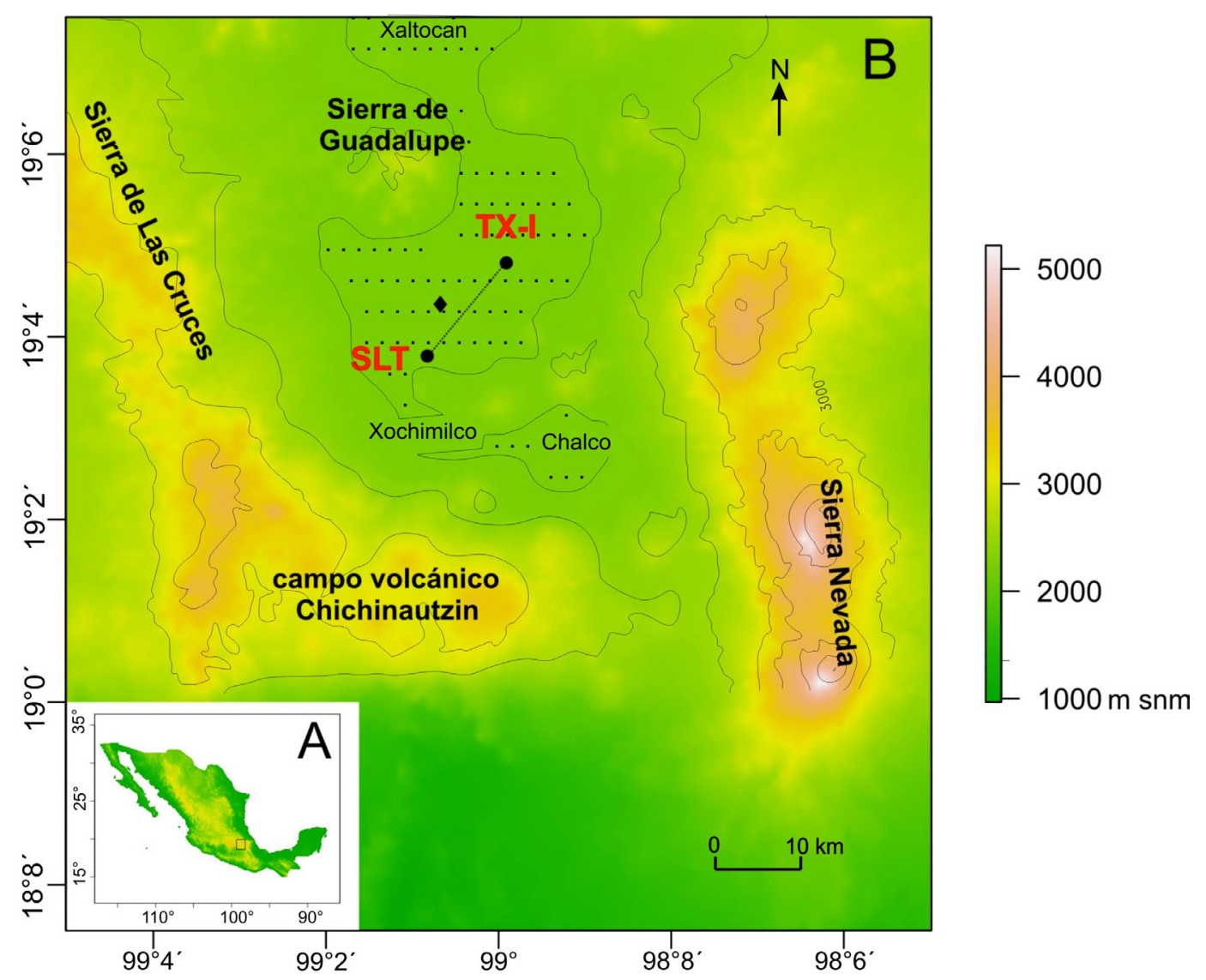

Figura 1. A) Mapa señalando la cuenca de México. B) Modelo de elevación de la cuenca de México donde se indica la ubicación de los pozos San Lorenzo Tezonco (SLT) (Arce et al., 2013) y Texcoco-I (TX-I) (Hiriart y Graue, 1969; Marsal y Graue, 1969). La línea punteada corresponde al límite de la zona lacustre de la cuenca. Aeropuerto de la Ciudad de México señalado por $\downarrow$.

de cierre de cuenca son las de Mooser et al. (1974) y UrrutiaFucugauchi y Martín del Pozzo (1993). Sin embargo, datos recientes de ${ }^{40} \mathrm{Ar} /{ }^{39} \mathrm{Ar}$ indican que el emplazamiento de este alto estructural inició hace $1.2 \mathrm{Ma}$, lo que causó el bloqueo hidrológico e inició la sedimentación lacustre en el sector central (lago de Texcoco) (Arce et al., 2013).

A partir de la perforación de varios pozos profundos, iniciando con el Texcoco-I (TX-I) en 1967 que alcanzó una profundidad de $2068 \mathrm{~m}$ (Oviedo de León, 1967) y continuando con la serie de pozos perforados después del sismo de 1985: Roma (3200 m), Mixhuca (2452 m), Tulyehualco-1 (3000 m), Copilco (2258 m) (Pérez-Cruz, 1988) y San Lorenzo Tezonco (SLT) (2008 m) recientemente perforado (Arce et al., 2013), se ha avanzado en el conocimiento de la geología de la cuenca. La presencia de calizas de edad Cretácica se han reportado en los pozos Mixhuca y Tulyehualco a $1581 \mathrm{~m}$ y $2059 \mathrm{~m}$ respectivamente (Pérez-Cruz, 1988). Por encima de dichas calizas, durante el Cenozoico hay grandes espesores de rocas volcánicas en todos los pozos y se han propuesto cuatro episodios volcánicos principales: a) vulcanismo del Oligoceno; b) coladas basálticas y andesíticas del Mioceno inferior $(23.3$ a $21.7 \mathrm{Ma})$; c) lavas andesíticas del Mioceno medio ( 15 a $\sim 9 \mathrm{Ma}$ ); d) lavas máficas del Pleistoceno al reciente (Ferrari et al., 2002).

En todos los pozos perforados en la cuenca se identifican espesores importantes de sedimentos lacustres dentro de los primeros $\sim 700 \mathrm{~m}$, mismos que varían en espesor. Los datos publicados del pozo Texcoco-I indican la existencia de un espesor de $482 \mathrm{~m}$ de arcillas lacustres (Hiriart y Graue, 1969; Marsal y Graue, 1969), $300 \mathrm{~m}$ para Mixhuca y ca. $100 \mathrm{~m}$ en los pozos Copilco y Roma (Pérez-Cruz, 1988). Para el pozo SLT se reportan sedimentos lacustres en los primeros $70 \mathrm{~m}$ y otra capa entre 590 a $604 \mathrm{~m}$ de profundidad (Arce et al., 2013) (Figura 2).

Las secuencias sedimentarias lacustres de la cuenca, en particular las que se encuentran en los primeros $30 \mathrm{~m}$, han sido objeto de estudio de varias disciplinas ya que contienen evidencias de las condiciones ambientales pasadas. Destacan las investigaciones que se han realizado analizando los paleoindicadores biológicos (polen, diatomeas, ostrácodos, etcétera) y los paleoindicadores no biológicos (propiedades magnéticas, isótopos, tefras, etcétera). Así, se ha documentado la evolución paleoambiental de los últimos 30000 años en los lagos de Chalco (Lozano-García et al., 1993; Caballero y Ortega-Guerrero, 1998; Lozano-García 
y Ortega-Guerrero, 1998, Ortega-Guerrero y Newton, 1998; Ortega-Guerrero et al., 2000; Ortega-Guerrero, 1992; Herrera-Hernández, 2011; Correa-Metrio et al., 2013), Texcoco (Bradbury, 1971, 1989) y al norte de la cuenca en el lago de Tecocomulco (Caballero et al., 1999; Roy et al.,

\section{Texcoco-1}
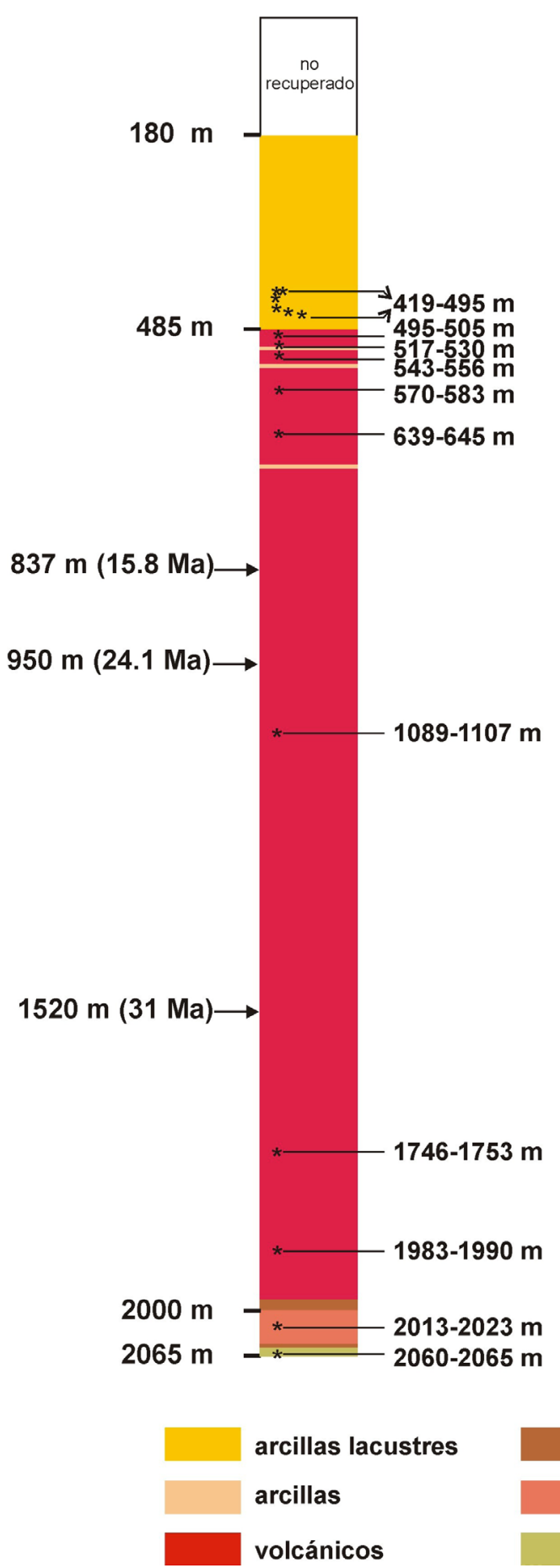

2008; Roy et al., 2009) a través del estudio de múltiples indicadores, destacando los cambios en la flora, niveles lacustres y cambios en procesos de erosión y actividad volcánica.

La presencia de espesores de arcillas lacustres en los

\section{San Lorenzo Tezonco}

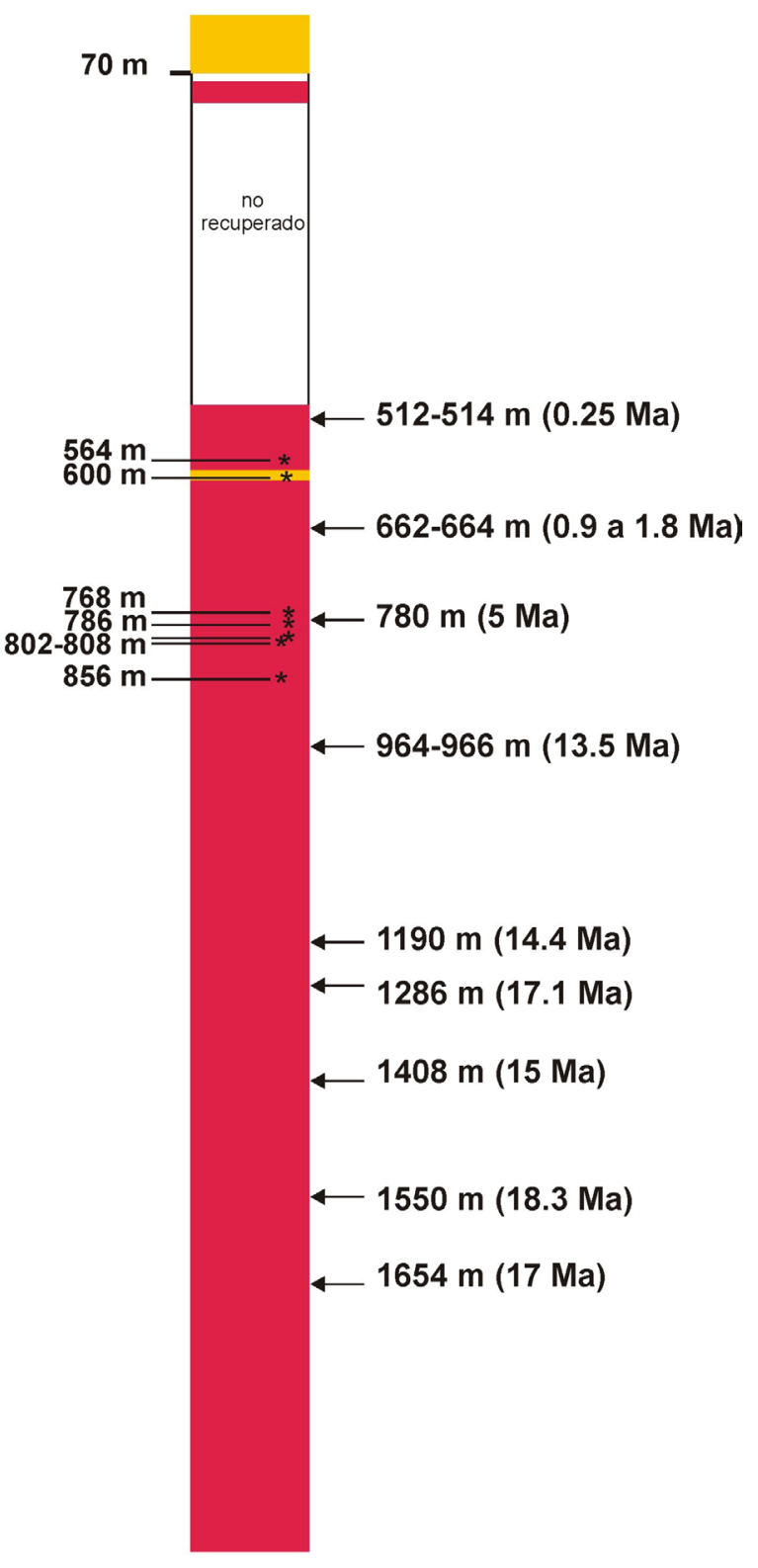

Figura 2. Columnas litológicas resumidas de los pozos Texcoco-I, las edades son las señaladas por Hiriart y Graue (1969), y San Lorenzo Tezonco con las edades reportadas por Arce et al. (2013). La muestras seleccionadas están indicadas por * y se señala la profundidad de las mismas. 
pozos profundos de la cuenca abre la posibilidad de conocer la composición y la evolución de la flora en edades previas al Pleistoceno tardío y Holoceno. En este trabajo son analizados los palinomorfos presentes en muestras de los pozos Texcoco-I y San Lorenzo Tezonco para documentar la paleoflora y su composición, y establecer la presencia de cuerpos de agua a través de palinomorfos indicadores de ambientes lacustres.

\subsection{Pozos profundos San Lorenzo Tezonco (SLT) y Texcoco-I (TX-I)}

El pozo SLT $\left(19^{\circ} 22^{\prime 4} 44.18 \mathrm{~N}\right.$; $99^{\circ} 05^{\prime \prime 05.10)}$ fue perforado en 2013 (Figura 2) usando el método rotatorio, resultando en la recuperación de muestras de canal (Morales-Casique et al., 2014). La secuencia volcanosedimentaria de SLT con $2008 \mathrm{~m}$ de profundidad está compuesta además de los sedimentos lacustres superiores por depósitos volcánicos como lavas y flujos piroclásticos, andesitas, dacitas e ignimbritas (Arce et al., 2013) (Figura 2). Varios horizontes fueron fechados y la correlación con eventos volcánicos y sus productos indica que la secuencia abarca $>18 \mathrm{Ma}$. La denominada Andesita antigua (1930 a $2008 \mathrm{~m}$ de profundidad) puede ser correlacionada con el vulcanismo del grupo Balsas del Eoceno, o con el campo volcánico de Huautla del Oligoceno (Arce et al., 2013).

El registro y descripción de la secuencia del pozo TX1, perforado en el antiguo lago de Texcoco (Figura 2), fue publicado por Oviedo de León (1967) en un informe del Instituto Mexicano del Petróleo y otros detalles del proyecto en Hiriart y Graue (1969) y Marsal y Graue (1969). Los primeros $182 \mathrm{~m}$ no fueron recuperados y a partir de esta profundidad hubo una recuperación continua de núcleos hasta los $954 \mathrm{~m}$. Posteriormente, la recuperación de núcleos fue intermitente, hasta alcanzar una profundidad total de 2065 m. La secuencia está conformada principalmente por rocas volcánicas como dacitas, ignimbritas, basaltos y brechas. En la base reportan la presencia de anhidrita y un conglomerado calcáreo de 2060 a $2065 \mathrm{~m}$ de profundidad (Oviedo de León, 1967) (Figura 2). Las edades de Potasio/ Argón disponibles en Hiriart y Graue (1969) indican que la secuencia del pozo TX-I abarca posiblemente del Eoceno superior/Oligoceno temprano al Pleistoceno.

\section{Métodos}

Con base en la litología reportada por Oviedo de León (1967) fueron seleccionadas 24 muestras de roca de varios núcleos del TX-I, y 7 muestras de canal del pozo SLT (Tabla 1). Para la extracción de palinomorfos se realizó el siguiente procedimiento: cada muestra de roca fue triturada utilizando un mortero de metal y las muestras de canal del SLT lavadas cuidadosamente para luego ser trituradas. Posteriormente, $50 \mathrm{~g}$ de cada muestra fueron colocadas en vasos de precipitado y se añadió $\mathrm{HCl}$ al $10 \%$ para la eliminación de los carbonatos presentes, posteriormente fueron lavadas hasta quedar neutras. El siguiente paso fue añadir HF para la eliminación de silicatos durante 24 a 48 hrs y luego lavadas hasta neutralizarlas. La materia orgánica fue eliminada con la aplicación de $\mathrm{KOH}$ y/o solución Shultz $\left(\mathrm{HNO}_{3}\right.$ y $\left.\mathrm{KClO}_{3}\right)$. En algunos casos las muestras fueron teñidas con Safranina. El residuo obtenido de cada muestra fue montado en HC (hidroacetilcelulosa), bálsamo de Canadá y gelatina glicerinada para su posterior observación al microscopio óptico. Las muestras fueron analizadas bajo el microscopio y se realizó el conteo de los palinomorfos y los microfósiles fueron fotografiados. Los diagramas palinológicos de abundancia fueron elaborados utilizando el software Tilia-Graph 2.1 (Grimm, 2004).

\section{Resultados}

De las 16 muestras procesadas del TX-1 (Tabla 1) todas resultaron positivas. Contabilizándose un total de 300 palinomorfos en todas las muestras, que corresponden a 34 palinomorfos distintos, de los cuales 27 fueron identificados a diferentes niveles taxonómicos. Un promedio de 18 palinomorfos por muestra se estimó para el TX-I, la muestra con mayor número de palinomorfos correspondió a la profundidad de $500 \mathrm{~m}$ y la más baja con 7 tipos a la profundidad de $577 \mathrm{~m}$ con 71 tipos de palinomorfos. Para el

Tabla 1. Listado de muestras analizadas de los pozos Texcoco-I y San Lorenzo Tezonco. * Muestra negativa

\begin{tabular}{lcc}
\hline Pozo TX-I & $\begin{array}{c}\text { Prof. } \\
\text { (m) }\end{array}$ & $\begin{array}{c}\text { Pozo SLT } \\
\text { Prof. (m) }\end{array}$ \\
\hline 7052 Núcleo 20, - caja 1-2 & $419-430$ & 564 \\
7058 Núcleo 21, - caja 1-2 & $430-443$ & 600 \\
7061 Núcleo 21, - caja 3 & $443-451$ & 768 \\
7090 Núcleo 22, - caja 1 & $451-456$ & 786 \\
7092 Núcleo 23, - caja 1 & $456-469$ & 802 \\
7105 Núcleo 25, - caja 4 & $482-495$ & 808 \\
7109 Núcleo 26, - caja 1-4-8 & $495-505$ & $856^{*}$ \\
7113 Núcleo 28, - caja 2-4 & $517-530$ & \\
7124 Núcleo 30, - caja 1 & $543-556$ & \\
7134 Núcleo 32, - caja 2 & $570-583$ & \\
7149 Núcleo 37, - caja 7 & $639-645$ & \\
7198 Núcleo 64, - caja 1 & $1089-1107$ & \\
7390 Núcleo 78, - caja 5-9 & $1746-1753$ & \\
7392 Núcleo 79, - caja 1-6 & $1983-1990$ & \\
7395 Núcleo 80, - caja 2 & $2013-2023$ & \\
7396 Núcleo 81, - caja 3-8 & $2060-2065$ & \\
\hline
\end{tabular}


SLT sólo seis de las siete muestras procesadas y analizadas fueron positivas con un total de 120 palinomorfos y 30 tipos polínicos. El promedio de palinomorfos por muestra fue de 20 , la muestra de $802 \mathrm{~m}$ presentó 35 tipos mientras que la más baja fue la de $768 \mathrm{~m}$ con 6 palinomorfos.

Ambos registros comparten 12 palinomorfos: Alnus, Picea, Pinus, Quercus, Amaranthaceae, Cupressaceae, Fabaceae, Poaceae, esporas de helechos tipo monolete y trilete, y las microalgas Botryococcus y Pediastrum (Figura 3).

Los conteos de palinomorfos de ambos sitios se presentan en forma de diagramas polínicos (Figuras 4 y 5). Los taxa más abundantes en ambas secuencias corresponden a polen de elementos arbóreos como Pinus, Quercus y Alnus. Otros taxa como Liquidambar, Carya, Anacardiaceae, Fabaceae y los tipos 4, 5 y 7 presentaron mayor abundancia en el TX-I. Dentro del conjunto de herbáceas destacan Amaranthaceae, Asteraceae y Poaceae con presencia de $<2$ granos de polen en el SLT, mientras que en el TX-I alcanzan valores de $>4$ granos de polen. Botryococcus y Pediastrum, microalgas indicadoras de cuerpos de agua, se recuperaron en las muestras de los primeros $600 \mathrm{~m}$ del TX-I y en las muestras de 600 y $804 \mathrm{~m}$ del SLT.

\section{Discusión}

\subsection{San Lorenzo Tezonco}

Para el pozo SLT, fueron fechados diez niveles por el método de ${ }^{40} \mathrm{Ar} /{ }^{39} \mathrm{Ar}$ y uno con $\mathrm{U} / \mathrm{Pb}$ en circones extraídos de la matriz rocosa, de acuerdo con estos fechamientos los depósitos cubren al menos los últimos $18 \mathrm{Ma}$ (Arce et al., 2013). Con base en las características litológicas y las edades disponibles Arce et al. (2013) proponen una correlación entre las secuencias volcano-sedimentarias de los pozos SLT y Tulyehualco. Dado que en la mayor parte de los pozos que han sido perforados en la cuenca de México hasta ahora, los depósitos lacustres están presentes en los primeros $\sim 700 \mathrm{~m}$ es posible suponer que esta sedimentación lacustre pudo iniciar hace al menos 1 Ma. Aunque la edad del depósito lacustre entre 590 y 604 $\mathrm{m}$ no se conoce, su edad puede ser acotada puesto que las lavas dacíticas $(604$ a $700 \mathrm{~m})$ que lo subyacen tienen una edad de 1 Ma y la secuencia andesítica basáltica (590 a 510 m) que la sobreyace tiene una edad de 250000 años. Los palinomorfos recuperados de la parte superior del pozo SLT muestran una composición similar a los conjuntos polínicos del Pleistoceno tardío (30000 a 50000 años). Los estudios sobre la paleoflora del Pleistoceno tardío en la cuenca de México han aportado información sobre la composición de las comunidades vegetales y los cambios asociados a la variabilidad climática y ambiental (Lozano-García et al., 1993; Lozano-García, 1996; Lozano-García y OrtegaGuerrero, 1994, 1998; Caballero et al., 2010; Sedov et al., 2010). Para este periodo, los registros paleoecológicos de los lagos de Chalco, Texcoco y Tecocomulco muestran la dominancia de varios tipos polínicos, principalmente polen de elementos arbóreos como son Pinus, Quercus, Alnus y Cupressaceae, mientras que el componente herbáceo generalmente está presente en los diagramas con polen de Poaceae, Asteraceae y Amaranthaceae. Los taxa más abundantes dentro del conjunto arbóreo de la parte superior del pozo SLT son Pinus, Quercus, Cupressaceae y Alnus. También se registraron polen de Picea y Ericaceae. En esta sección del registro el elemento herbáceo está compuesto por Poaceae, Asteracae, Ambrosia y Amaranthaceae.

La presencia de algas clorofítas Botryococcus y Pediastrum, confirma la existencia de un lago para este tiempo también sugerido por la litología reportada por Arce et al. (2013). Con base en las edades del pozo SLT la muestra de $604 \mathrm{~m}$ corresponde al Pleistoceno temprano.

Considerando las edades del pozo SLT, las muestras de 768 a $808 \mathrm{~m}$ son de edad pliocénica. Hasta ahora, poco se conoce sobre la paleoflora pliocénica de México, destacan las investigaciones en la zona de Santa María Amajac (Arellano-Gil et al., 2005; Velasco de León y OrtizMartínez, 2010) donde se reportan macrorestos de varias especies de Quercus, Populus, Cercocarpus (Rosaceae), Rhus (Anacardiaceae) y Poaceae; en cuanto a microrestos (polen y esporas) se documenta polen de Pinus, Picea, Alnus, Quercus, Poaceae, Asteraceae, Amaranthaceae, Ambrosia, Lamiaceae, Pediastrum y Botryococcus (CruzHernández, 2012). Además, Graham (1976) presenta la composición de la flora pliocénica de la Formación Paraje en Veracruz. Los microfósiles de SLT fueron muy escasos en los niveles de 768 y $784 \mathrm{~m}$, para la muestra de $768 \mathrm{~m}$, sólo se recuperó Arcella, una tecameba que actualmente se desarrolla en lagos, suelos y pantanos, además granos de polen porados sin identificar. A los $784 \mathrm{~m}$ se recuperó polen de Pinus y tres tipos polínicos no identificados. Sin embargo, en las muestras más profundas (802 y 808 m) el registro de microfósiles fue mayor con Pinus, Alnus, Quercus y algunas hierbas como Amaranthaceae, Poaceae y Lamiaceae. En general los taxa presentes en las muestras pliocénicas de SLT ya han sido previamente documentadas en otros estudios, en particular en los sedimentos del paleolago de Santa María Amajac donde se reconstruye para el Plioceno la existencia de entre otras asociaciones de plantas, bosques de pino encino y bosques mesófilos (Arellano-Gil et al., 2005; Velasco de León y Ortiz-Martínez, 2010; CruzHernández, 2012) lo que muestra cómo los elementos de la flora moderna ya estaban presentes desde el Plioceno en la región central de México.

También se registró Botryococcus en SLT a $802 \mathrm{~m}$ de profundidad, sugiriendo la existencia de un cuerpo de agua. Temporalmente esta muestra se encuentra en los niveles fechados entre $5 \mathrm{Ma}(780 \mathrm{~m})$ y $13.5 \mathrm{Ma}(964-966 \mathrm{~m})$ (Arce et al., 2013). Para este periodo Ferrari et al. (2002) proponen la existencia de un hiatus en la actividad volcánica del final del Mioceno al Plioceno inferior en la cuenca de México. Estas muestras están dentro de la unidad hidrogeológica IV 

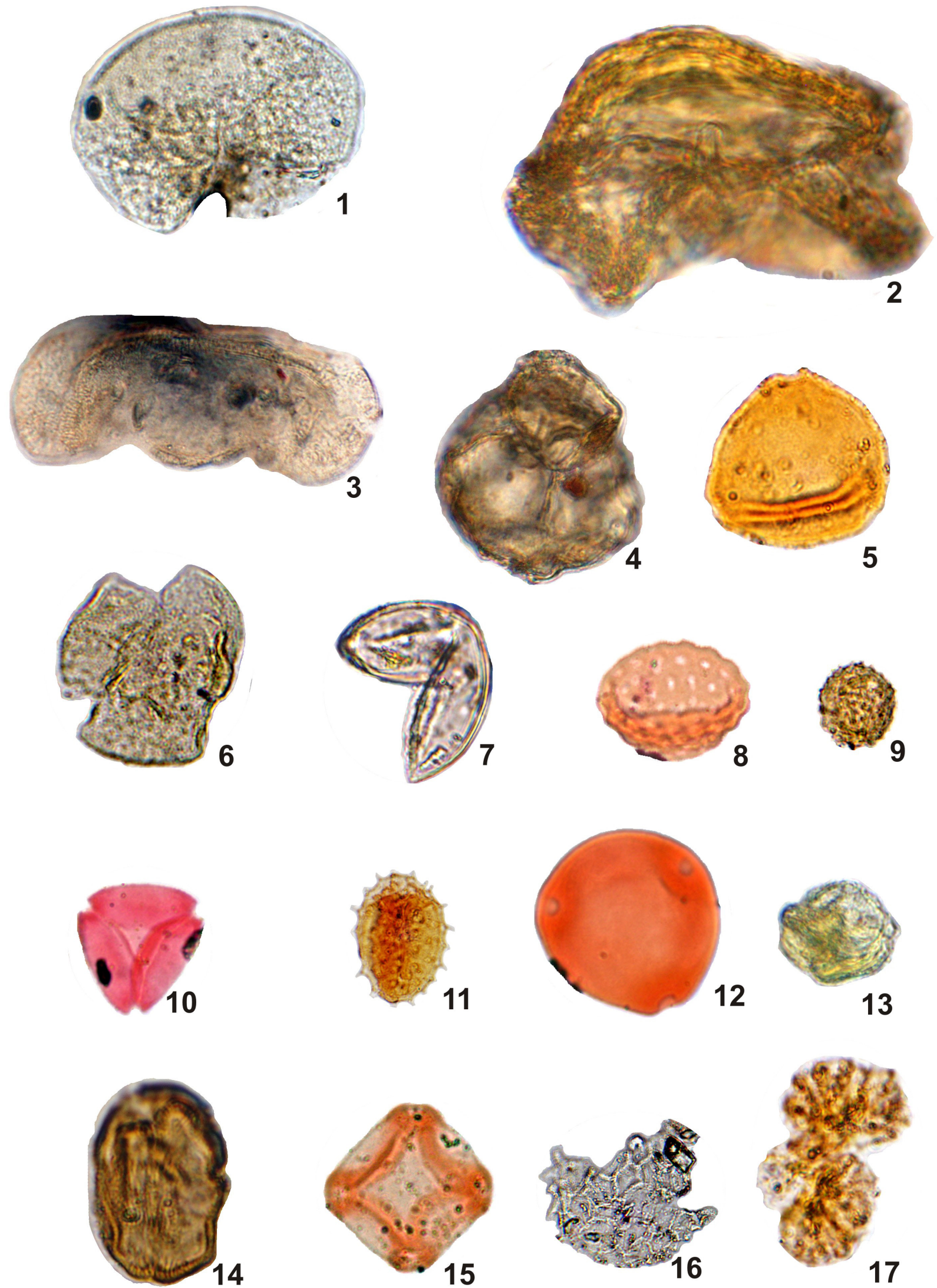

Figura 3. Microfotografías (1000X) de algunos palinomorfos recuperados de muestras de los pozos San Lorenzo Tezonco y Texcoco-I. 1. Picea, 2. Abies, 3. Pinus, 4. Ericaceae, 5. Engelhardtia, 6. Quercus, 7. Cupressaceae, 8. Amaranthaceae, 9. Ambrosia, 10. Myrtaceae, 11. Asteraceae, 12. Carya, 13. Drosera, 14. Tipo estriado, 15. Alnus, 16. Pediastrum, 17. Botryococcus. 


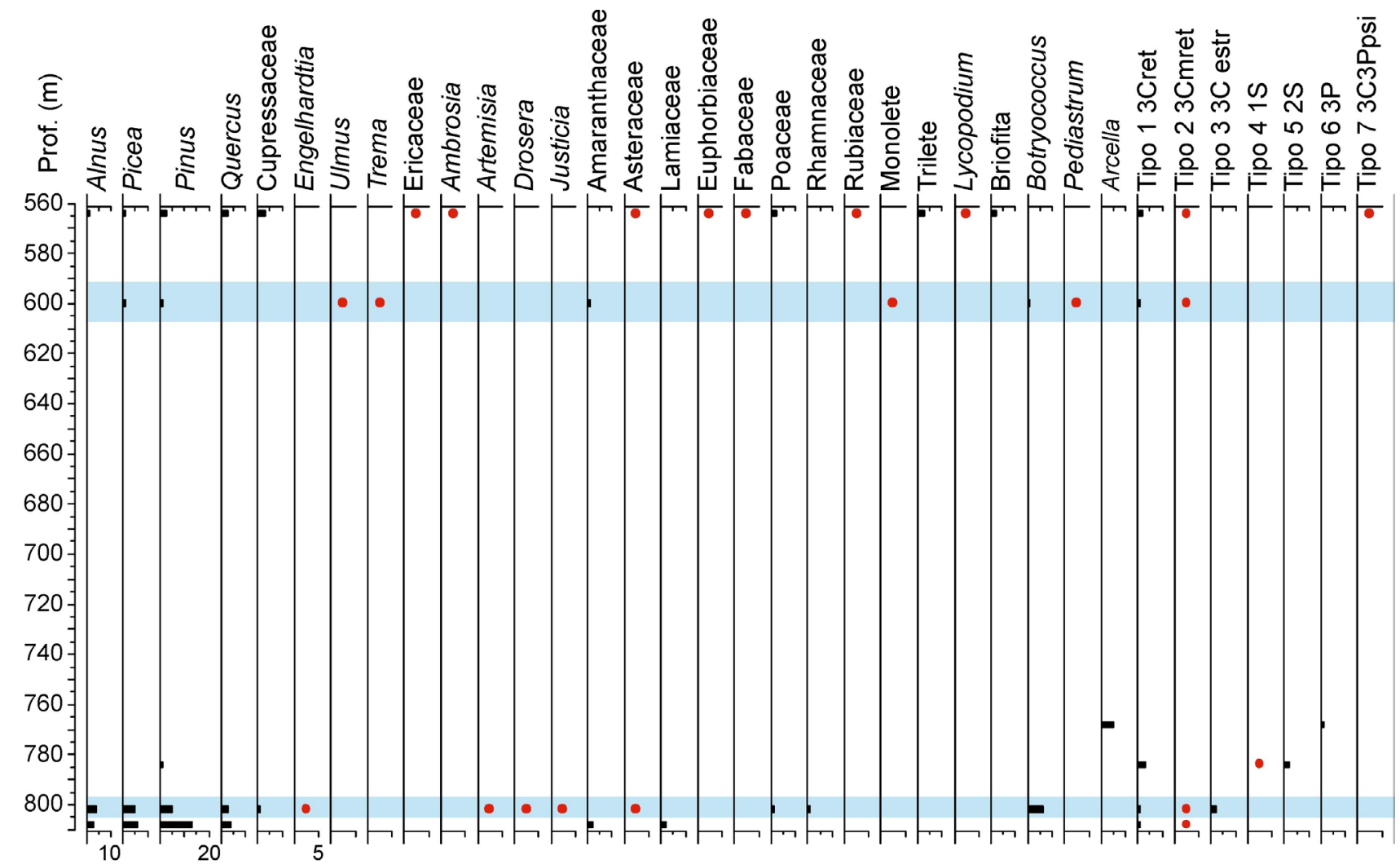

Figura 4. Diagrama palinológico de presencia correspondiente a las muestras del pozo San Lorenzo Tezonco indicando con el cuadro azul la posible presencia de un cuerpo de agua. La presencia del taxon (1 grano) se señala con un punto rojo.

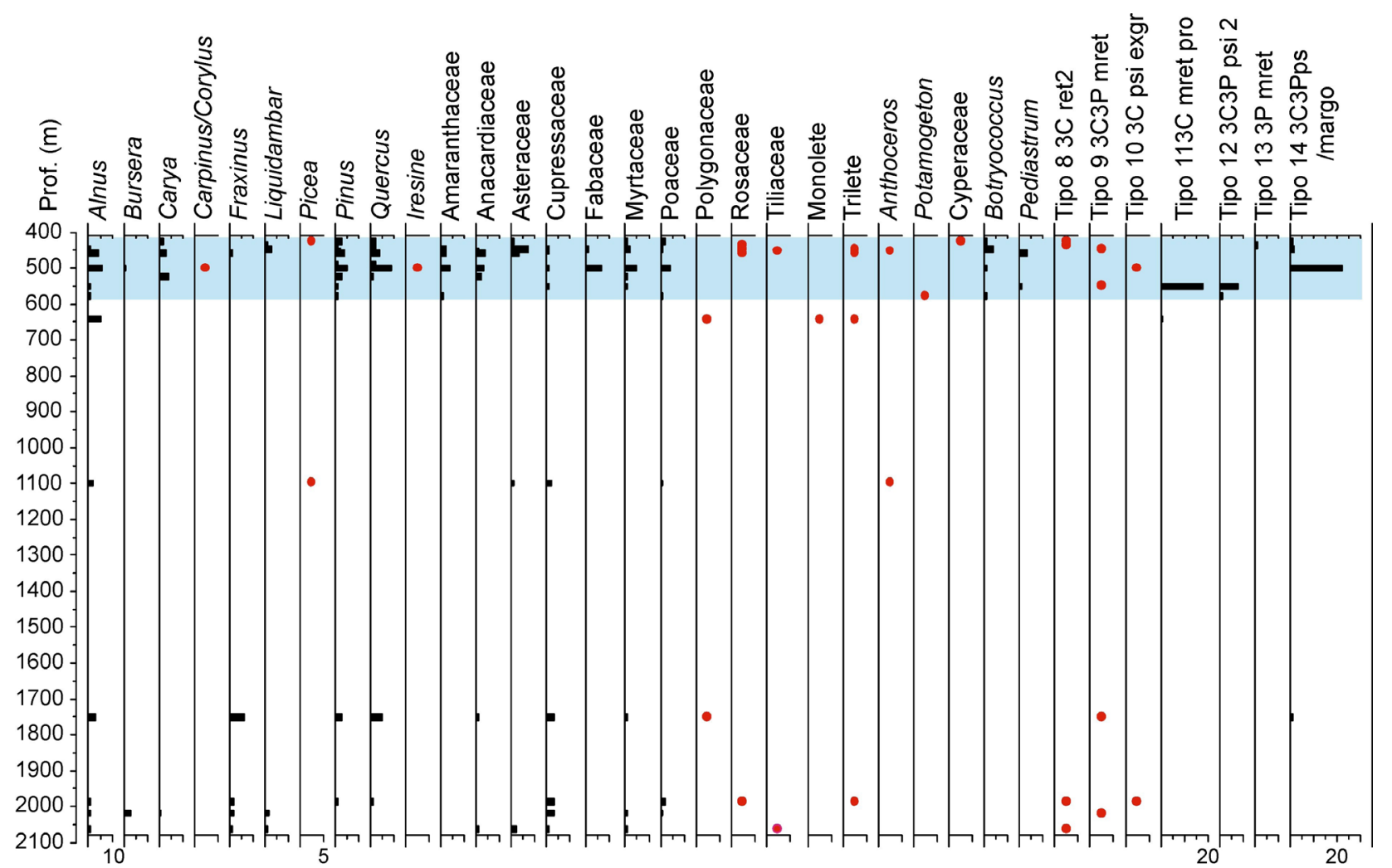

Figura 5. Diagrama palinológico de presencia del pozo Texcoco-I indicando con el cuadro azul la posible presencia de un cuerpo de agua. La presencia del taxon (1 grano) se señala con un punto rojo. 
definida por Morales-Casique et al. (2014) donde infieren la presencia de arcillas por el comportamiento hidráulico. Teniendo en consideración lo anterior, es factible que durante esta época de disminución en la actividad volcánica, se haya desarrollado un lago en la cuenca de México, como lo indican los microfósiles. Sin embargo, los datos que aquí se presentan provienen de muestras de canal y para confirmar este hallazgo es necesario contar con núcleos continuos para tener control sobre profundidad y edad.

\subsection{Texcoco-I}

Para las muestras de 425 a $642 \mathrm{~m}$ del pozo TX-I es posible que el rango de edad sea Plioceno-Pleistoceno, ya que la composición de su microflora es similar a lo reportado en las comunidades del Pleistoceno tardío. Sin embargo, hay algunas diferencias como la abundante presencia de Myrtaceae y Anacardiaceae y dos tipos no identificados. En cuanto a los elementos correspondientes a las comunidades lacustres se registraron Potamogeton, una planta acuática, Cyperaceae, la cual se desarrolla en ambientes húmedos, Anthoceros y también se recuperaron las microalgas Botryococcus y Pediastrum; todos los elementos de este conjunto de microfósiles son indicadores de la existencia de un lago.

La sección de 1098 a 1986 m del pozo TX-I puede tener una edad del Oligoceno considerando los fechamientos de Potasio/Argón (Hiriart y Graue, 1969). Los estudios paleobotánicos que establecen la composición de las floras eocénicas y oligocénicas en México son escasos. Para Tepexi de Rodríguez se reporta por Martínez-Hernández y Ramírez-Arriaga (2006) una microflora variada de rocas inicialmente definidas como de edad eocénica, pero posteriormente fueron fechadas por Silva-Romo (2010) quien les asigna una edad del Oligoceno (32 Ma). En esta sección del pozo TX-I (1098 a 1986 m) la composición y abundancia de los taxa es distinta a los conjuntos PlioPleistocénicos. Destacan la ausencia de Carpinus-Corylus, Iresine, Amaranthaceae y Fabaceae, así como por el conjunto de acuáticas/subacuáticas del registro. Picea, Pinus, Quercus, Alnus no son tan abundantes en particular en la muestra más profunda $(1986 \mathrm{~m})$, mientras que Liquidambar, Fraxinus y Cupressaceae alcanzan hasta 6 granos de polen. Algunos de los taxa como Bursera, Carya, Carpinus-Corylus, Liquidambar, Pinus, Quercus, Picea, Amaranthaceae, Anacardiaceae, Asteraceae, Cupressaceae, Mytaceae, Poaceae, Polygonaceae, Rosaceae, Tiliaceae y Anthoceros ha sido registradas en palinofloras oligocénicas de Tepexi de Rodríguez (Martínez-Hernández y RamírezArriaga, 2006).

Destaca en la parte basal del TX-I (2018 y 2063 m) la ausencia de Pinus, Quercus y Picea sugiriendo posiblemente la existencia de condiciones poco adecuadas para estos taxa como podrían ser los ambientes cálidos y húmedos con alta estacionalidad, lo que explicaría la presencia de anhidrita en la base del pozo, indicativa de alta evaporación. Es probable que para ese tiempo, la futura cuenca de México fuera una cuenca baja, localizándose a una menor altura, a diferencia de la cuenca moderna que esta a $>$ de 2000 msnm resultado del levantamiento asociado al vulcanismo. Además, la composición litológica de la muestra más profunda (2063 m) del pozo TX-I se caracteriza por un conglomerado calcáreo que puede ser contemporáneo al Grupo Balsas de edad Eoceno (Molina-Garza y Ortega-Rivera, 2006). Ambos hechos sugieren que la microflora de esta sección del pozo TX-I pudiera tener una edad del Eoceno tardío. Hay, sin embargo, pocos estudios palinológicos del Eoceno en México aunque como señalan Dávalos-Álvarez et al. (2007), el género Pinus conjuntamente con la presencia de Picea son los taxa dominantes de los conjuntos polínicos del Oligoceno al Pleistoceno en México. La ausencia de Pinus, Picea y Quercus en la parte profunda el TX-1 pudiera implicar que se trate del Eoceno tardío, pero es necesario contar con más fechas para el pozo TX-1.

\section{Conclusiones}

La utilización del análisis palinológico para determinar la flora cenozoica de la cuenca de México a través del estudio de muestras provenientes de los pozos SLT y TX-I aportó información relevante sobre la composición de ésta. Los resultados muestran la presencia de varios géneros comunes en las asociaciones de plantas modernas, sin embargo, la abundancia de éstos varió a lo largo del tiempo y entre ambas secuencias. La ausencia de Pinus, Quercus y Picea en la base del pozo TX-I sugiere que estos conjuntos palinológicos probablemente tienen una edad del Eoceno tardío, además, su composición indica la existencia de climas cálidos/húmedos con estacionalidad marcada. Con los microfósiles indicadores de cuerpos de agua como son las microalgas Botryococcus y Pediastrum, fue posible identificar periodos de depositación lacustre en la parte superior de ambas secuencias correspondiente a edades estimadas del Plioceno-Pleistoceno.

\section{Agradecimientos}

Esta investigación se realizó con apoyo del proyecto UNAM DGAPA IN109012. Agradecemos al Dr. José Luis Arce y a Dr. Oscar Escolero quienes nos proporcionaron las muestras del pozo de San Lorenzo Tezonco. Los autores agradecemos los comentarios y sugerencias de tres revisores anónimos que contribuyeron a mejorar el trabajo.

\section{Referencias}

Arce, J.L., Layer, P.W., Morales-Casique, E., Benowitz, J., Rangel, E., Escolero, O., 2013, New constraints on the subsurface geology of the Mexico City Basin: The San Lorenzo Tezonco deep well, on the basis of ${ }^{40} \mathrm{Ar}{ }^{39} \mathrm{Ar}$ geochronology and whole-rock chemistry: Journal 
of Volcanology and Geothermal Research, 266, 34-49.

Arellano-Gil, J., Velasco-de Léon, P., Silva-Pineda, A., Salvador-Flores, R., Beltrán-Romero, F., 2005, Origen y características geológicas del paleo-Lago de Amajac, Hidalgo: Revista Mexicana de Ciencias Geológicas, 22(2), 199-211.

Bradbury, J.P., 1971, Paleolimnology of lake Texcoco, Mexico. Evidence from diatoms: Limnology and Oceanography, 16, 180-200.

Bradbury, J.P., 1989, Late Quaternary lacustrine paleoenvironments in the Cuenca de México: Quaternary Science Reviews, 8, 75-100.

Caballero, M., Ortega-Guerrero, B., 1998, Lake Levels since about 40000 Years Ago at Lake Chalco, near Mexico City: Quaternary Research, 50, 69-79.

Caballero, M., Lozano-García, S., Ortega-Guerrero, B., UrrutiaFucugauchi, J., Macias J.L., 1999, Environmental characteristics of lake Tecocomulco, northern basin of Mexico, for the last ca. 50000 years: Journal of Paleolimnology, 22, 399-411.

Caballero, M., Lozano-García, S., Vázquez-Selem, L., Ortega-Guerrero, B., 2010, Evidencias de cambio climático y ambiental en registros glaciales y en cuencas lacustres del centro de México durante el último máximo glacial: Boletín de la Sociedad Geológica Mexicana, 62, 359-377.

Correa-Metrio, A., Bush, M., Lozano-García, S., Sosa-Nájera, S., 2013, Millennial-Scale temperature change velocity in the continental neotropics, PLoS ONE, 8(12), e81958.

Cruz-Hernández, J.M., 2012, Estudio palinológico de sedimentos pliocénicos de Santa María Amajac, Hidalgo durante el Neógeno: México D.F., Universidad Nacional Autónoma de México, tesis de licenciatura, $64 \mathrm{p}$.

Dávalos-Álvarez, O.G., Nieto-Samaniego, A.F., Alaniz-Álvarez, S.A., Martínez-Hernández, E, Ramírez-Arriaga, E., 2007, Estratigrafía cenozoica de la región de Tehuacán y su relación con el sector norte de la falla de Oaxaca: Revista Mexicana de Ciencias Geológicas, $24,197-215$.

Ferrari, L., Mena, M., López Martínez, M., Albarrán, J., Silva-Romo, G., Mendoza-Rosales, C., González-Cervantes, N., 2002, Estratigrafía y tectónica de la cuenca de la ciudad de México y áreas colindantes: Geos, 22, 150.

Graham, A., 1976, Studies in Neotropical Paleobotany II, The Miocene communities of Veracruz, Mexico: Annals of the Missouri Botanical Garden, 63, 787-842.

Grimm, E., 2004, Tilia and TGView 2.0.2. Illinois State Museum. Research and Collection Center, Springfield, Illinois.

Herrera-Hernández, D., 2011, Estratigrafía y análisis de facies de los sedimentos lacustres del Cuaternario tardío en la cuenca de Chalco: México, D.F., Universidad Nacional Autónoma de México, tesis de maestría, $122 \mathrm{p}$.

Hiriart, F., Graue, R., 1969, Proyecto Texcoco, Nabor Carrillo: El hundimiento de la ciudad de México proyecto Texcoco, Secretaría de Hacienda y Crédito Público, México, 328 p.

Lozano-García, M.S., 1996, La vegetación Cuaternaria en el Centro de México: Registros Palinológicos e Implicaciones Paleoclimáticas: Boletín de la Sociedad Botánica de México, 58, 113-127.

Lozano-García, S., Ortega-Guerrero, B., 1994, Palynological and magnetic susceptibility records of Chalco lake, central Mexico: Palaeogeography, Palaeoclimatology, Palaeoecology, 109, 177-191.

Lozano-García M.S., Ortega-Guerrero, B., 1998, Late Quaternary environmental changes of the central part of the Basin of Mexico; correlation between Texcoco and Chalco basins: Review of Paleobotany and Palynology, 99, 77-93.

Lozano-García, S., Ortega-Guerrero, B., Caballero-Miranda M., Urrutia-Fucugauchi, J., 1993, Late Pleistocene and Holocene paleoenvironments of the Chalco lake, Central Mexico: Quaternary Research, 40, 332-342.

Marsal, R.J., Graue, R., 1969, El subsuelo del Lago de Texcoco, Nabor Carrillo: El hundimiento de la ciudad de México proyecto Texcoco: México, Secretaría de Hacienda y Crédito Público, 328 p.
Martínez-Hernández, E., Ramírez-Arriaga, E., 2006, Tertiary palynofloristics correlations between Mexican formation with emphasis in dating the Balsas group, en Vega, F., Nyborg, T., Perrilliat, M.C., Montellano-Ballesteros, M., Cevallos-Ferriz, S.R.S., Quiroz-Barroso, S. (eds.), Studies on Mexican Paleontology: Dordrecht, Springer, 24, 20-42.

Molina-Garza, R.S., Ortega-Rivera, A., 2006, Cronostratigraphy and paleomagnetism of the Balsas Group in the Tuzantlán-Copalillo basin, northern Guerrero state, Mexico: Revista Mexicana de Ciencias Geológicas, 23(2), 215-232.

Morales-Casique, E., Escolero, O.A., Arce, J.L., 2014, Resultados del pozo San Lorenzo Tezonco y sus implicaciones en el entendimiento de la hidrogeología regional de la cuenca de México: Revista Mexicana de Ciencias Geológicas, 31(1), 64-75.

Mooser, F., Nair, A.E.M., Negendank, J.F.W., 1974, Paleomagnetic investigations of Tertiary and Quaternary igneous rocks; VII, A paleomegnetic and petrologic study of volcanics of the Valley of Mexico: Geologische Rundschau, 63, 451-483.

Ortega-Guerrero, B., 1992, Paleomagnetismo, magnetoestratigrafía y paleoecología del Cuaternario tardío en el lago de Chalco, cuenca de México: México D. F., Universidad Nacional Autónoma de México, tesis doctoral, $161 \mathrm{p}$.

Ortega-Guerrero, B., Newton, A.J., 1998, Geochemical Characterization of Late Pleistocene and Holocene Tephra Layers from the Basin of Mexico, Central Mexico: Quaternary Research, 50, 90-106.

Ortega-Guerrero, B., Thompson, R., Urrutia-Fucugauchi, J., 2000, Magnetic properties of lake sediments from Lake Chalco, central Mexico, and their palaeoenvironmental implications: Journal of Quaternary Science, 15, 127-140.

Oviedo de León, A., 1967, Estudio Geológico del Subsuelo, Basado en los Datos Obtenidos de la Perforación del Pozo Profundo Texcoco No. 1: México D.F., Instituto Mexicano del Petróleo, reporte técnico, 95 p.

Pérez-Cruz, G., 1988, Estudio sismológico de reflexión del subsuelo de la Ciudad de México: México D.F., Universidad Nacional Autónoma de México, tesis de maestría, $83 \mathrm{p}$.

Roy, P.D., Caballero, M., Lozano, R., Smykatz-Klossd, W., 2008, Geochemistry of late quaternary sediments from Tecocomulco lake, central Mexico: implication to chemical weathering: Chemie der erde-geochemistry, 68, 383-393.

Roy, P.D., Caballero, M., Lozano R., Pi T., Morton O., 2009, Late Pleistocene-Holocene geochemical history inferred from Lake Tecocomulco sediments, Basin of Mexico, Mexico: Geochemical Journal, 43, 49-64.

Sedov, S., Lozano-García, M.S., Solleiro-Rebolledo, E., McClung de Tapia, E., Ortega-Guerrero, B., Sosa-Nájera, S., 2010, Tepexpan Revisited: A multiple proxy of local environmental changes in relation to human occupation from a paleolake shore section in central Mexico: Geomorphology, 122(3-4), 309-322.

Silva-Romo, G., 2010, Origen tectónico y evolución de la cuenca Tehuitzingo-Tepexi estado de Puebla: México D.F., Universidad Nacional Autónoma de México, tesis doctoral, 209 p.

Urrutia-Fucugauchi, J., Martín del Pozzo, A.L., 1993, Implicaciones de los datos paleomagnéticos sobre la edad de la Sierra de Chichinautzin, cuenca de México: Geofísica Internacional 32, 523-533.

Velasco de León, P., Ortiz-Martínez, E., 2010, Nuevas especies de Quercus en el Plioceno de Santa María Amajac, Hidalgo, México: Revista Mexicana de Ciencias Geológicas, 27(2), 264-277.

Manuscrito recibido: Mayo 16, 2014

Manuscrito corregido recibido: Diciembre 5, 2014

Manuscrito aceptado: Febrero 2, 2015 